\title{
Frequency Dependent Model of Leakage Inductance for Magnetic Components
}

\author{
Peter R. Wilson ${ }^{1}$ and Reuben Wilcock ${ }^{1}$ \\ ${ }^{1}$ School of Electronics and Computer Science, University of Southampton, UK \\ *corresponding author, E-mail: prw@ecs. soton. ac.uk
}

\begin{abstract}
Theoretical methods for the calculation of winding losses with respect to frequency have been well documented, but the variation in leakage inductance of magnetic components due to frequency has been less well addressed. In this paper Dowell's well-known theoretical approach is tested using measurements and finite element analyses. The results presented show deficiencies in the accuracy of models generated using Dowell's approach and highlight the tradeoffs between model complexity, simulation time and accuracy when the finite element analysis method is applied. An alternative behavioral model is presented which models the frequency variation in leakage inductance accurately, is easy to characterize from simple measurements or calculations and is robust.
\end{abstract}

\section{Introduction}

Extensive theoretical and empirical research has taken place to establish the variation of winding losses with frequency. Dowell [1] provides a theoretical framework for the calculation of losses in transformer windings, and this has been developed by a large number of authors since including Cheng and Evans [2],[3]. Roberts, Mathys and Schauwers [4] have studied the accuracy of the theory and have noted discrepancies between the theoretical and empirical values of losses obtained while Niemela et al [5] have extended the theory for general multiple winding topologies.

There has been much less effort applied to the equivalent theoretical calculation of leakage inductance, especially at higher frequencies where eddy current and proximity effects become significant. The modeling of the leakage inductance of magnetic components and its variation with frequency has been addressed theoretically by Dowell [1] and by Dauhajre [6], Dauhajre \& Middlebrook [7] and Hsu, Middlebrook and Cuk [8]. Of these, however, only Dowell provides a theoretical approach for the estimation of the combined eddy current and proximity effects and the resulting leakage inductance variation with frequency. More recent work by Hurley and Wilcox [9] has provided an alternative approach, but is specifically for toroidal transformers. The problem with all of these methods is the inaccuracy of the predicted values for leakage inductance as the frequency increases.

In general, the focus of the effort to characterize the high frequency behaviour of windings, in particular leakage inductance, has been concentrated on the application of finite element analysis, with Silvester \& Konrad [10], Tabaga, Pierratt \& Blache [11], Skutt, Lee, Ridley \& Nicol [12], Dai \& Lee [13]-[14] and Cherpentier, Lefevre \& Lajoie-Mazenc [15] demonstrating the approach. The methodology is now well understood and is often provided as an application example in commercial finite element analysis software packages.

The development of the finite element analysis software as the integral part of a procedure to calculate the high frequency behavior of magnetic components has been developed extensively by Asensi et al [16]-[18] and Prieto [19]-[21]. This procedure has the function of providing models for practical electric circuit simulation of power applications, and this has also been addressed by Okyere \& Heinemann [22] and Evans \& Heffernan [23]. Unfortunately the procedure requires exhaustive finite element analyses of the transformer models, which become more finely meshed as the frequency increases thereby either increasing the simulation time required, or reducing the accuracy of the result. It is not uncommon for a simple transformer to require several hours of finite element analysis to obtain the leakage inductance variation with frequency. The resulting model obtained may also be cumbersome and exhibit convergence difficulties. Lopera et al [26] and Pernia et al [27],[28] have developed a one-dimensional approximation of the behaviour of the magnetic component windings including frequency variations. This has been developed into a software package UO-M2T by Prieto et al [29] that produces a netlist model of the magnetic component for use in circuit simulation. There are two issues to consider with this type of approach. The first is the size and complexity of the resulting model. If the model consists of a large number of elements, then this may impact on the convergence and time of the circuit simulation. The second issue is the decoupling of the model elements from the original physical geometry. If a model can be constructed which retains this link, then sensitivity of key parameters such as interlayer gap or winding size can be usefully investigated without the requirement for a remodeling of the device. This technique is discussed specifically in this paper.

In this paper, the difficulties of both the theoretical and numerical methods will be addressed from a practical perspective to provide a useful circuit simulation model in a reasonable time. Assessments are made of the theoretical models and various finite element analysis modeling 
techniques with respect to measured results to enable an informed choice to be made for the appropriate level of model required. Finally a resulting model structure is proposed which models the leakage inductance variation with frequency, and can be characterized with a relatively small number of measurements or finite element analyses.

\section{Evaluation of Dowell's Equations}

If the theoretical equations of Dowell [1] and Dauhajre et al [6]-[7] are evaluated, it can be shown that the low frequency leakage inductance is effectively obtained from the same equation, the apparent differences are purely a matter of nomenclature. If the two-winding transformer shown in figure 1 is taken as an example, Dauhajre provides the expression for the leakage inductance based on this geometry in (1).

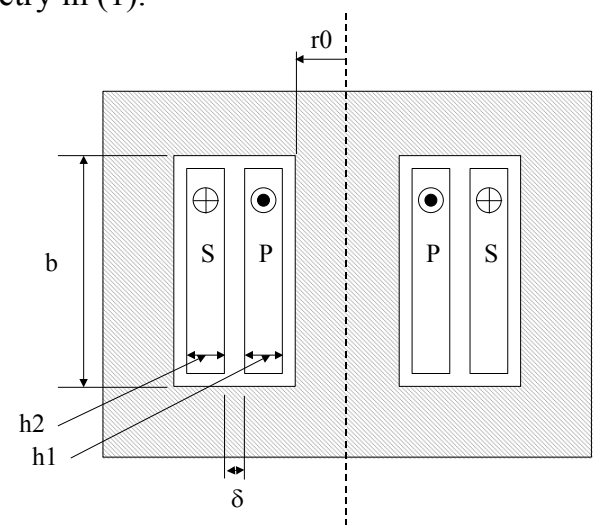

Figure 1: Basic Transformer Winding Structure

$$
l=2 \pi \mu_{0} N_{1}^{2} \frac{1}{b}\left\lfloor\begin{array}{l}
h_{1}\left(\frac{r_{0}}{3}+\frac{h_{1}}{4}\right) \\
+\delta\left(r_{0}+h_{1}+\frac{\delta}{2}\right) \\
+h_{2}\left(\frac{r_{0}+h_{1}+\delta}{3}+\frac{h_{2}}{12}\right)
\end{array}\right\rfloor
$$

This approach is fundamentally the same as Dowell's in that the energies stored in each winding space and the interwinding spaces are calculated independently and then combined to provide the complete leakage inductance value, referred to a single winding (e.g. the primary). Dauhajre stops at this point, but Dowell makes the point that by dividing the energy regions into winding and spaces, the winding regions are frequency dependent, due to eddy currents and proximity effects, but the spaces are not. For a two winding transformer, the overall expression for the leakage inductance is given from Dowell's equations by (2), where $L_{w l}$ is the leakage inductance in winding $1, L_{w 2}$ is the leakage inductance in winding $2, F_{L 1}$ and $F_{L 2}$ are the frequency variations in the leakage indutances of winding 1 and 2 respectively, $L_{g}$ is the leakage inductance due to the interwinding gap and $L_{U}$ is the leakage inductance due to the interlayer gaps.

$$
L_{\text {leakage }}=F_{L 1} L_{w 1}+F_{L 2} L_{w 2}+L_{g}+L_{U}
$$

Where the frequency variation of each winding leakage inductance is represented by a lumped equation for all of the eddy current and proximity effects as shown by (3)-(6).

$$
F L=\left\{\frac{3 M^{\prime \prime}+\left(m^{2}-1\right) D^{\prime \prime}}{m^{2}\left|\alpha^{2} h^{2}\right|}\right\}
$$

Where $M^{\prime \prime}$ is the imaginary part of the function $M$ given in (4), and $D$ " is the imaginary part of the function $D$ given in (5). $\alpha$ is the expression for effective skin depth given in (6), $m$ is the number of winding layers and $h$ is the layer thickness.

$$
\begin{aligned}
& \boldsymbol{M}=\alpha \boldsymbol{h} \operatorname{coth} \alpha \boldsymbol{h} \\
& \boldsymbol{D}=2 \alpha \boldsymbol{h} \tanh \frac{\alpha \boldsymbol{h}}{2} \\
& \alpha=\sqrt{\frac{\boldsymbol{j} \boldsymbol{w} \mu_{0} \eta}{2}}
\end{aligned}
$$

To assess the accuracy of these equations, a 2 winding transformer was constructed using a Phillips RM12-3F3 core, and wound using 24 SWG (0.559 mm diameter) enameled copper wire with both the primary and secondary having 2 layers of 21 turns each. The inter-winding gap was measured to be $1.2 \mathrm{~mm}$. The leakage inductance was obtained by measuring the impedance across the primary, while short-circuiting the secondary winding, varying the frequency from $100 \mathrm{~Hz}$ to $1 \mathrm{MHz}$. The short circuit wires on the secondary winding were kept to a minimum length to reduce the introduction of extraneous leakage due to the measurement set up. The correlation of the measured and calculated leakage inductance values at $1000 \mathrm{~Hz}$ seems to indicate the shorting wires have little impact on the measured leakage inductance. The low frequency leakage inductance was calculated using Dauhajre's equation (1) and verified using Dowell's approach with the value estimated at $12.52 \mathrm{uH}$. A Mathcad model was then created for equation (3) and the packing factor $(\eta)$ varied using 1.0 (perfect winding area coverage), 0.866 (perfect round conductor packing) and 0.5 (practical estimate). The resulting variation of measured and theoretical values is given in figure 2 .

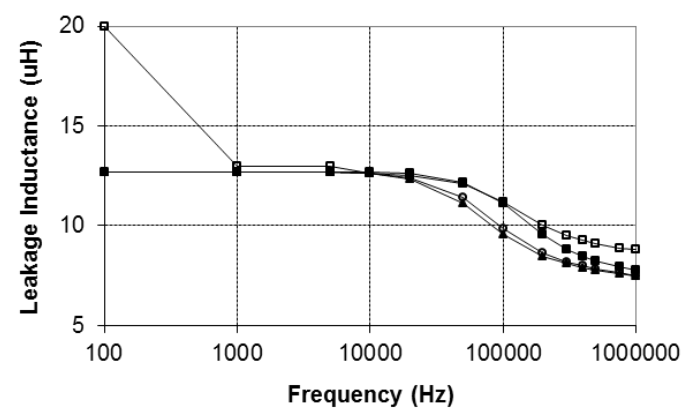

Measured $\rightarrow-$ Dowell $n=0.5 \multimap$ Dowell $n=1.0 \multimap$ Dowell $n=0.866$ 
Figure 2: Theoretical and empirical leakage inductance variation with frequency

There are two interesting points to note from figure 2 . The first is that as the frequency drops to around $100 \mathrm{~Hz}$, the apparent leakage inductance seems to increase. The explanation for this, is that as the frequency drops, the impedance of the magnetizing inductance falls and becomes comparable to the winding resistance. This has the consequence that the measured inductance can no longer be assumed to be an accurate measure of the leakage inductance alone. This causes the measured leakage inductance to apparently increase. The second point to notice is that as the frequency increases, the predicted leakage inductance decreases more quickly than the measured value. Niemela et al [5] and Robert et al [4] have also observed this difference. It is also noticeable that as the frequency tends to $1 \mathrm{MHz}$, the leakage inductance flattens off to a high frequency value. In summary, therefore, it is clear that Dowell's theory is not always accurate at higher frequencies at predicting the roll off of the leakage inductance, or its final high frequency value. This is probably due to the analysis by Dowell being effectively carried out in one dimension and not considering two dimensional effects.

\section{Estimation of Leakage using FEA}

The use of finite element analysis (FEA) for the estimation of leakage inductance has long been a standard technique, but there is a trade-off between the simulation time (depending on the complexity of the model) and the ultimate accuracy of the parameter derived. A study of the common model approximations used in the estimation of high frequency parameters was carried out, to establish the accuracy and simulation times for the example transformer previously used. In all cases an axis-symmetric approximation was used. Asensi et al [17] explain a procedure for applying two dimensional finite element analysis techniques to extract the parasitic parameters for magnetic components of the type used in this paper.

\subsection{Simple 2D Approximation with Lumped Windings}

The first model uses the approximation that the complete winding can be modeled in a $2 \mathrm{D}$ finite element analysis as a single block. The resulting model is given in figure 3 . The core model for 3F3 was modeled as a linear material with effective permeability 1583 (estimated from the Phillips datasheet).

The leakage inductance, referred to the primary winding can be estimated by applying equal and opposite field strengths to the primary and secondary windings (which if the number of turns are equal means equal and opposite currents). The resulting energy stored is effectively the energy stored in the winding area from which the leakage inductance can be directly calculated. The leakage inductance obtained using this model is shown in figure 4 , along with the measured results and the values derived by Dowell for a packing factor of 0.5 .

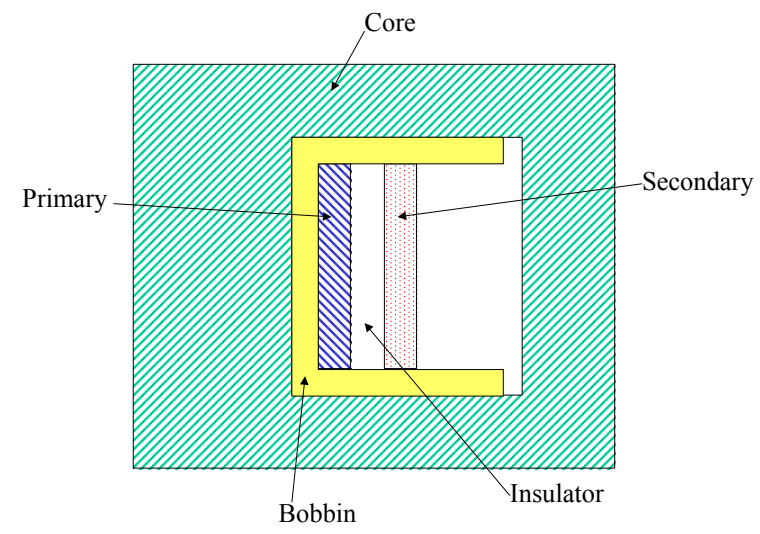

Figure 3: Simple 2D Transformer Model

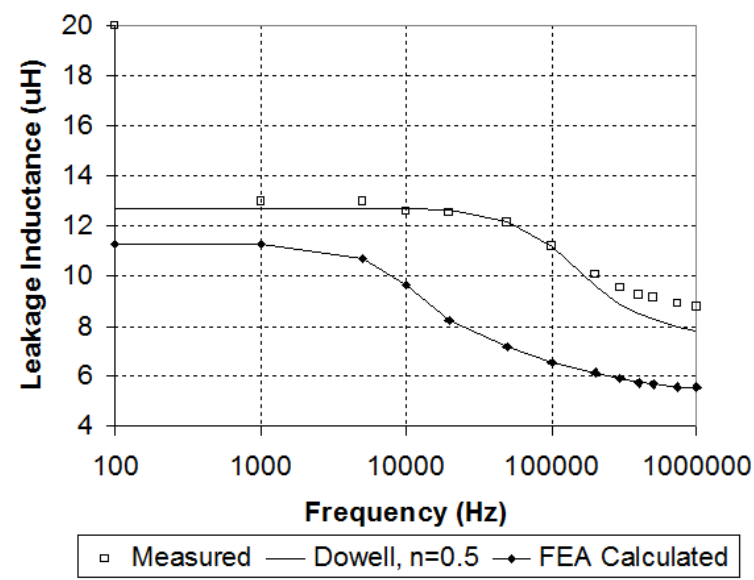

Figure 4: Simple 2D Finite Element Model

It is clear that this model does not accurately predict either the absolute value, or the frequency variation of the leakage inductance. However, it does show a less dramatic falling off of the leakage inductance with frequency than Dowell. The simple model of the winding does mean that the skin effect will be modeled differently than for individual turns, so that is not unexpected. This highlights that this commonly assumed model is clearly not adequate for accurate characterization of the parameters. At $1 \mathrm{MHz}$, the leakage inductance calculated using the finite element analysis was $37 \%$ below the measured value.

\subsection{Individual Layer 2D approximation}

The second model lumped each layer as a separate entity in the finite element analysis, so in this case there are 4 discrete layers, with the correct diameter based on the wire gauge. The resulting model is given in figure 5. The same tests were applied as for the simple $2 \mathrm{D}$ model, and the resulting leakage inductances shown in figure 6 . As the complexity of the models increase, the corresponding simulation times also increase so fewer analyses were carried out. 


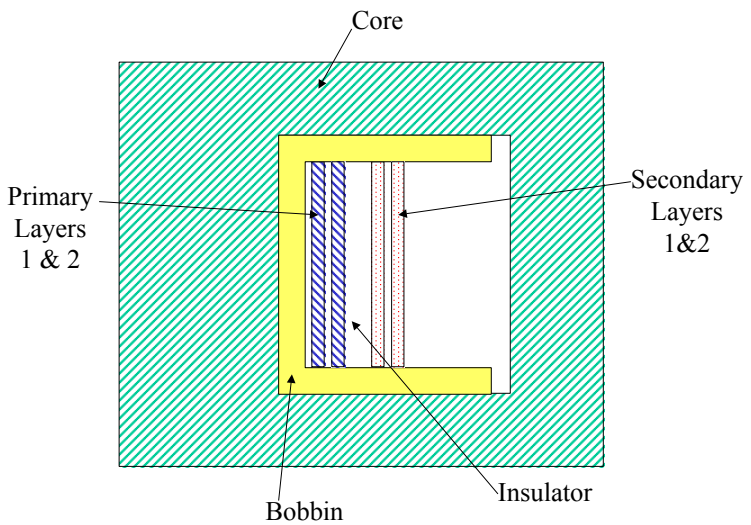

Figure 5: 2D model using individual Layers

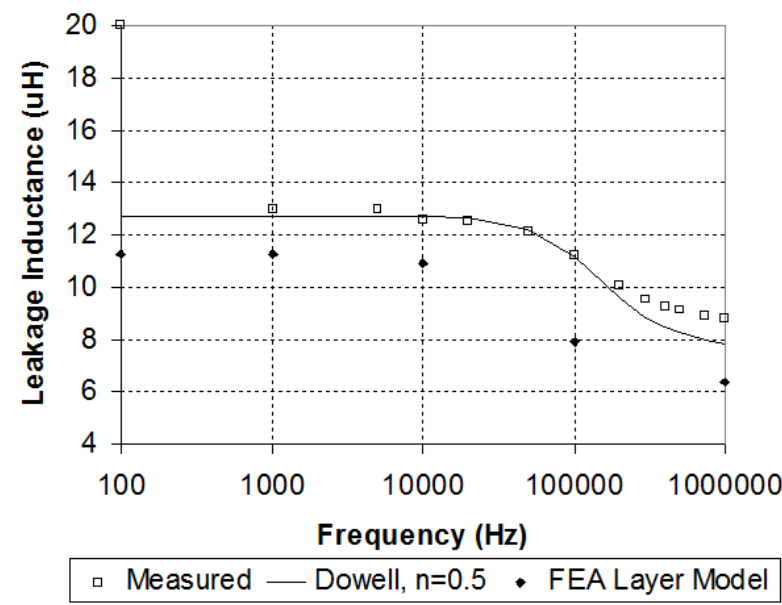

Figure 6: Layer by Layer 2D Finite Element Model

Using this model, the low frequency error was similar to that obtained using the simple 2D model, but the errors have decreased by about $10 \%$. At $1 \mathrm{MHz}$, the finite element calculated value of leakage inductance is $28 \%$ below the measured value.

\subsection{Turn by Turn 2D Model}

The final model is the most complex, and requires the modeling of each individual turn of the windings in the finite element model. The finite element model used is shown in figure 7.

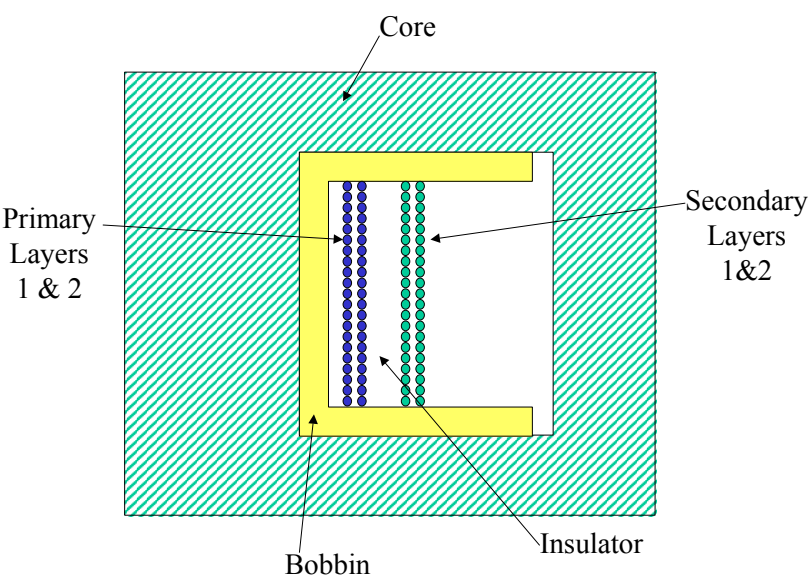

Figure 7: Turn by Turn 2D Model

The previous tests were carried out on this model, with a significant increase in simulation time observed. It was noticeable in this model that as the frequency increased, the simulation time increased dramatically from 33 minutes at $100 \mathrm{~Hz}$ to over 2 hours for a $1 \mathrm{MHz}$ analysis. The resulting leakage inductance results and measured values are shown in figure 8 .

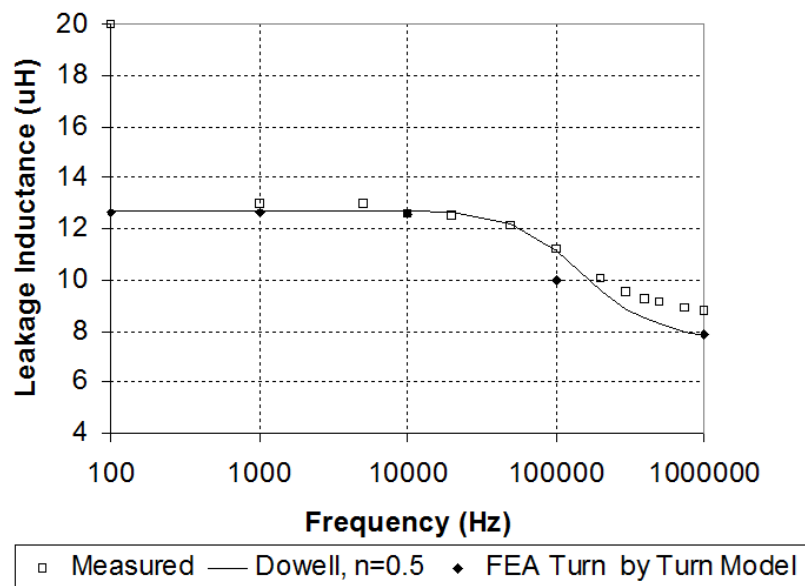

Figure 8: Turn by Turn 2D Finite Element Model

The results using this model are significantly better than the simpler 2D models, but at a heavy computational cost. To characterize this component using this approach would take many hours of simulation. The resulting error at $1 \mathrm{MHz}$ of leakage inductance of $-10 \%$ is reasonable, given the tolerances in the materials and geometries.

\section{Applying existing 1D Modeling Software}

Using a 1D approach to extract a detailed model of magnetic components has been well described by Lopera et al [26] and Pernia et al [27],[28] and implemented in the $\mathrm{UOM}^{2} \mathrm{~T}$ 
software described by Prieto et al [29]. This software was used to generate a spice model of the RM12 transformer, with the resulting leakage inductance variations as shown in figure 9. The results were obtained using the built in analyzer, which creates and runs a PSPICE model and test circuit, then returns the required values of leakage inductance or winding resistance.

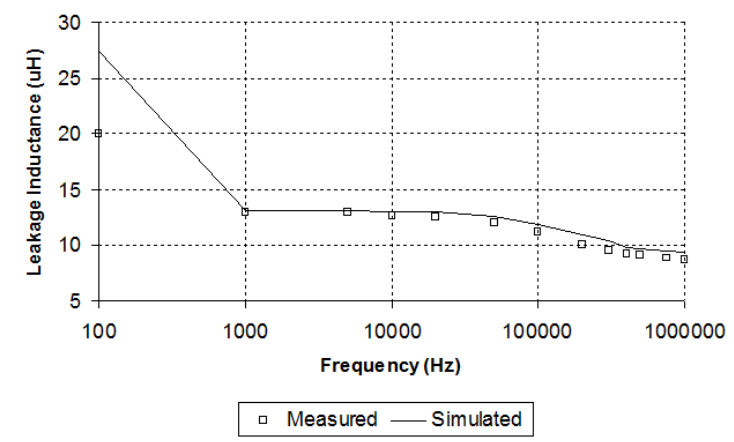

Figure 9: UOM2T Model Leakage Inductance Calculation

The comparison between measured and simulated results is good, but the resulting spice model in this case consisted of 71 elements. While this technique is extremely useful for designing the topology of devices, the resulting model is fairly cumbersome and does not provide the ability to vary parameters directly, without repeating the modeling procedure.

\section{Proposed new model for leakage inductance}

Given the deficiencies in both the theoretical and finite element analysis approaches demonstrated previously, there is the requirement for a simple model of the frequency variation of leakage inductance, which can reasonably accurately (to within 10\%) model the behaviour of the leakage inductance with frequency which can be characterized with either a few measurements or finite element analyses. A further requirement of the model was to provide a simple approach that would allow direct understanding of the effect specific model parameters would have on the circuit performance with minimal simulation overhead. A new model structure is therefore proposed which reasonably models the low and high frequency behaviour of leakage inductance, and the transition between these two states. If the model of a transformer is considered in the magnetic domain, as shown in figure 10, the component consists of two windings, and a magnetic circuit containing the core and leakage reluctances.

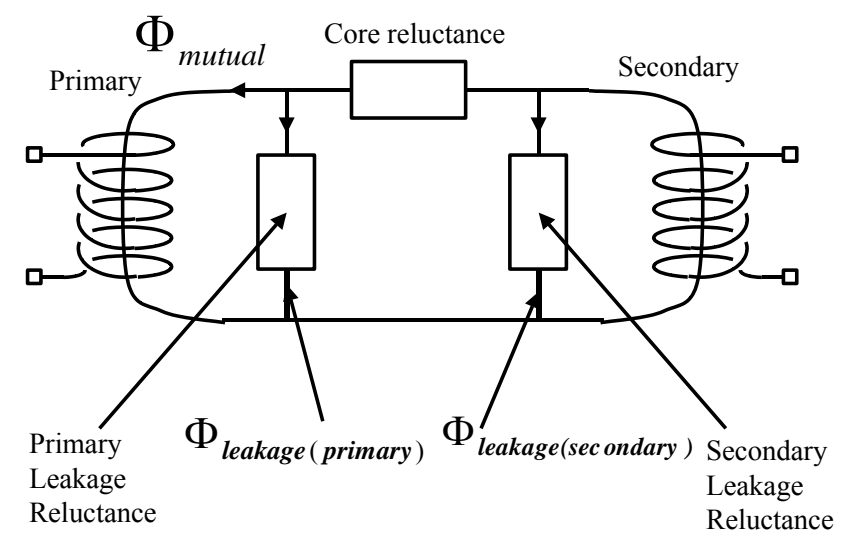

Figure 10: Electro-Magnetic Transformer Model

It is proposed to split each leakage reluctance into two parts, a d.c. or low frequency Reluctance $\left(\mathfrak{R}_{d c}\right)$ and a high frequency Reluctance $\left(\mathcal{R}_{h f}\right)$. At low frequencies the total reluctance is the parallel combination of $\mathfrak{R}_{d c}$ and $\mathfrak{R}_{h f}$ while at high frequencies the effect of the d.c. reluctance is reduced by eddy currents represented by the inductance $L_{\text {Loss }}$, so the total reluctance tends towards the high frequency value $\mathfrak{R}_{h f}$. The inductance $L_{\text {Loss }}$ in the magnetic domain is equivalent to a closed turn linked to the flux as described by Cherry [24] and Laithwaite [25]. The time constant of the $\mathcal{L}_{d c}$ reluctance and the loss inductor is effectively obtained from the conductor diameter. The resulting model of leakage reluctance is given in figure 11.

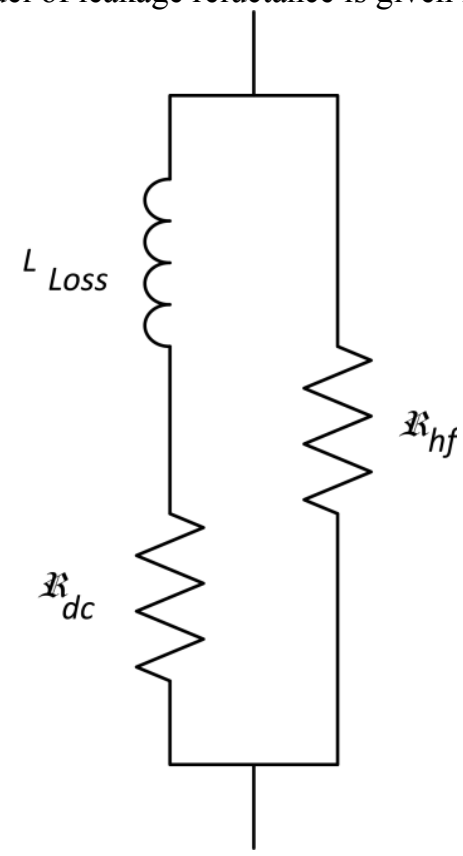

Figure 11: Modified model of leakage reluctance

This model can also be transformed into an equivalent in the electrical domain [24] using the configuration shown in figure 12 . 


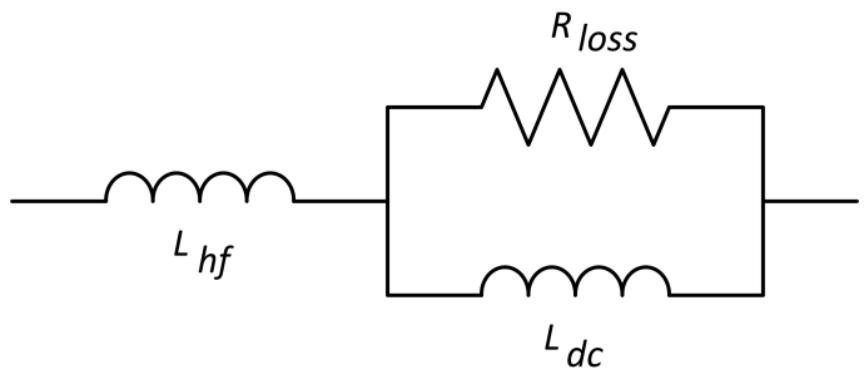

Figure 12: Proposed Leakage Inductance Model in the electrical domain

The $L_{\text {Loss }} \& \mathfrak{R}_{d c}$ (or $R_{\text {Loss }} \& L_{d c}$ using the electrical model) time constant can be estimated by using the frequency at which the skin depth is 0.5 the radius of the conductors used. This gives a reasonable estimate of the cut off frequency from which the loss component can be calculated. The low and high frequency leakage reluctances (or the inductances in electrical domain) can be measured or calculated using a high frequency and low frequency test thus reducing the number of tests (or finite element analyses) drastically. An alternative theoretical approach is to use Dauhajre's or Dowell's equations described in detail previouslt in this paper which separate the frequency and non-frequency dependent sections of the leakage inductance. Using this approach, the proportion of the leakage inductance due to the high frequency effect can be estimated.

The model of the transformer was constructed in SPICE as shown in figure 13, using the estimate of time constant based on the skin depth at half the conductor radius and the comparison with the measured leakage inductance given in figure 14. The ideal form of transformer model was used, with a measured magnetizing inductance of $10 \mathrm{mH}$ and perfect coupling $(k=1)$. The low frequency leakage inductance was measured at $13 \mathrm{uH}$, and the eventual effective high frequency leakage inductance was measured at $8.8 \mathrm{uH}$. Thus $L_{h f}$ was set to $8.8 \mathrm{uH}$ and $L_{d c}$ was set to the difference between the low and high frequency leakage inductance measurements, giving a value of $4.2 \mathrm{uH}$. In this model, the parasitic components were split evenly between the two windings, although the same value of leakage would be obtained by referring the values to one winding. The winding resistance $R_{w}$ was measured using a resistance bridge. The estimation of the $R_{\text {loss }}$ resistance, made with the skin depth at half the conductor radius, which can be estimated for 24 s.w.g. wire to be a depth of $0.13975 \mathrm{~mm}$, occurring at $223.6 \mathrm{kHz}$. Using this frequency, and the previously measured leakage inductance of $13 \mathrm{uH}$, the $R_{\text {loss }}$ value is estimated to be $2.9 \Omega$ using a simple RL time constant calculation. While this gives a reasonable estimate of the frequency variation, it is also the case that with such a simple model the accuracy of the final result may not be always optimal. It is an advantage of the simple model however, that improvements can be made in the matching with measured results by small variations in the value of $R_{\text {loss }}$. By including the magnetizing inductance and winding resistances in the model, the correct low frequency behaviour was also observed. The test circuit shown in figure 13 used a current source on the primary side, and effectively shorted out the secondary winding using a $1 \mathrm{~m} \Omega$ resistor. The imaginary part of the input voltage vin was then divided by $2 \pi f$ to give the effective leakage inductance shown in figure 14. The magnetizing inductance was obtained by measurement, with a value of $10 \mathrm{mH}$.
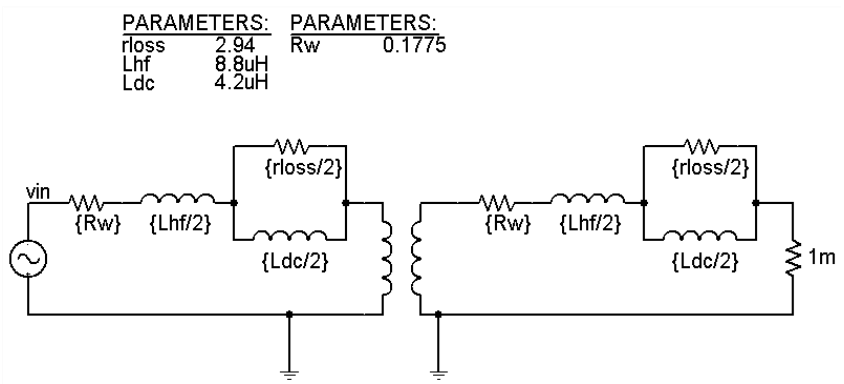

Figure 13: Test Circuit for transformer, including the modified leakage inductance model, implemented in Spice

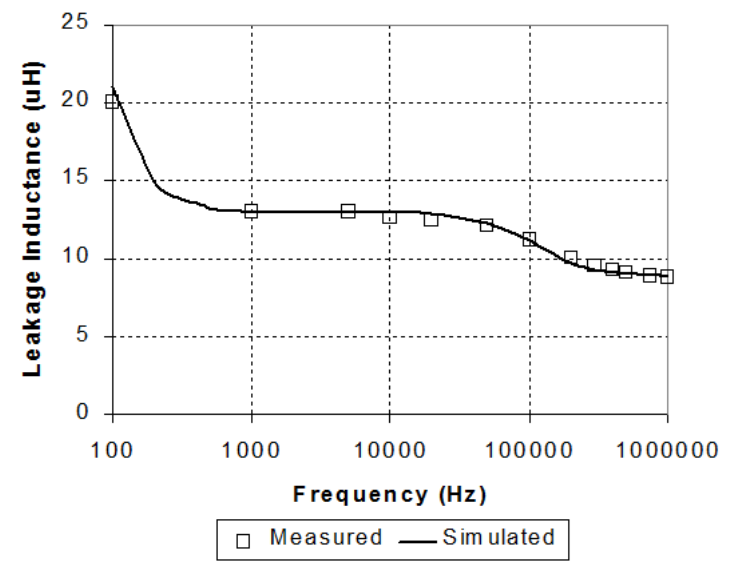

Figure 14: Measured and Simulated leakage Inductance for RM12 transformer

To demonstrate the technique more generally, another core was constructed, this time using an RM10-3F3 core, with two windings, each of 32 turns wound over two layers. The wire was 24 s.w.g as in the previous example. The resulting measured and simulated variations of leakage inductance with frequency are given in figure 15. The component values used in this case were $L_{h f}=3.72 \mathrm{uH}, L_{d c}=4.58 \mathrm{uH}$, $R_{w}=0.125 \Omega$ and $R_{\text {loss }}=3.5 \Omega$. The magnetizing inductance in this example was measured at $4.2 \mathrm{mH}$. 


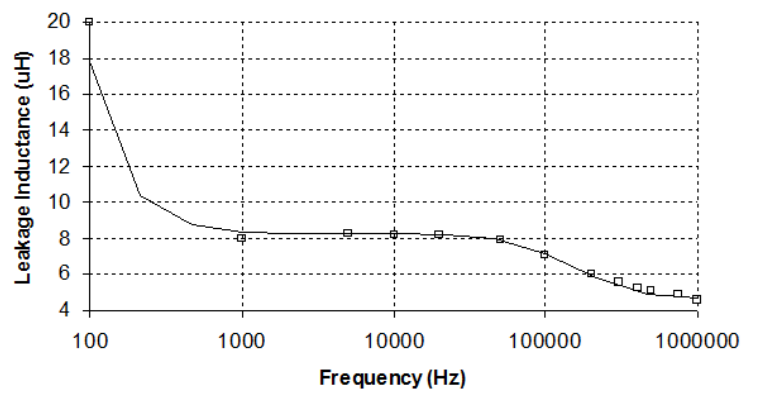

Measured - Simulated

Figure 15: Measured and Simulated leakage Inductance for RM10 transformer

\section{Conclusions}

Testing of Dowell's theory of the variation of leakage inductance with frequency has demonstrated limitations in the accuracy of the method at high frequencies. Study of the finite element analysis approaches to the calculation of leakage inductance has also highlighted difficulties in the practical application of this method to the effective calculation of leakage inductance variation with frequency in a reasonable time.

A detailed review of both the theoretical and numerical methods commonly used have shown deficiencies in the resulting predictions, and the proposed model structure in this work has been presented which accurately models the variation of leakage inductance with frequency using a simple approach. The resulting model is simple to characterize using measured or simulated values, stable and efficient to simulate.

\section{References}

[1] P. Dowell, "Effects of eddy currents in transformer windings", Proceedings of the IEE, Vol. 113, No 8, 1966, p1387

[2] K.W.E. Cheng \& P.D. Evans, Calculation of winding losses in high frequency toroidal inductors using single strand conductors, IEE Proceedings - Electric Power Applications, Vol. 141, No 2, Mar 1994, pp52-62

[3] K.W.E. Cheng \& P.D. Evans, Calculation of winding losses in high frequency toroidal inductors using multistrand conductors, IEE Proceedings - Electric Power Applications, Vol. 142, No 5, Sep 1995, pp313-322

[4] F. Robert, P. Mathys \& J.P. Schauwers, "Ohmic Losses Calculation in SMPS Transformers: numerical study of Dowell's approach accuracy", IEEE Transactions on Magnetics, Vol 34, No. 4, July 1998, pp1255-7

[5] Niemela V.A., Skutt G.R., Urling A.M., Chang Y.N., Wilson T.G., Owen H.A., Wong R.C., "Calculating the short-circuit impedance of a multiwinding transformer from its geometry", IEEE Power Electronics Specialists Conference (PESC), Vol 2, 1989, p 607-617
[6] A. Dauhajre, "Modeling and Estimation of Leakage Phenomena in Magnetic Circuits", $\mathrm{PhD}$ Thesis, California Institute of Technology, Pasadena, California, 1986.

[7] A. Dauhajre \& R.D. Middlebrook, Modeling and estimation of leakage phenomenon in magnetic circuits, IEEE Power Electronics Specialists Conference (PESC), 1986, pp 213-226

[8] S.P. Hsu , R.D. Middlebrook \& S. Cuk, "Transformer modeling and design for leakage control", Advances in Switched-Mode Power Conversion, 2nd Edition, TESLAco, 1983, Chapter 13, pp205-218.

[9] Hurley W.G., Wilcox D.J., "Calculation of Leakage Inductance in Transformer Windings", IEEE Transactions on Power Electronics, Vol. 9, No. 1, January 1994, pp121-126

[10]P.P. Silvester \& A. Konrad, Analysis of transformer leakage phenomena by high order finite elements, IEEE PES, 1973, p1843

[11] S. Tabaga, L. Pierrat \& F. Blache, "Parameter Computation of a planar transformer by 3D Finite Element Method", EPE Conference Proceedings, Seville, 1995, pp 1.273-276

[12] G. Skutt, F.C. Lee, R. Ridley \& D. Nicol, "Leakage Inductance and Termination Effects in a High Power Planar Magnetic Structure", IEEE Applied Power Electronics Conference and Exposition - APEC, Vol. 1, 1994, pp 295-301

[13]Dai N., Lee F.C., "Characterization and Analysis of Parasitic Parameters and their effects in Power Electronics Circuit", IEEE Annual Power Electronics Specialists Conference (PESC), Vol. 2, (1996), pp 1370-1375

[14]Dai N., Lee F.C., "High-Frequency Eddy-Current Effects in Low-Profile Transformer Windings", IEEE Annual Power Electronics Specialists Conference, v 1, (1997), pp 641-647

[15] Charpentier J.F., Lefevre Y., Lajoie-Mazenc M., “A 2D Finite Element Formulation for the study of the high frequency behaviour of wound components", IEEE Transactions on Magnetics, Vol. 32, No. 3, May 1996, pp 1098-1101

[16]R. Asensi, J.A. Cobos, O. Garcia, R. Prieto \& J Uceda, New Modeling Strategy for high frequency transformer windings, IECON Proceedings, Vol. 1, (1995), pp 246251

[17]R. Asensi, J.A. Cobos, O. Garcia, R. Prieto \& J Uceda, A full procedure to model high frequency transformer windings, IEEE Power Electronics Specialist Conference Proceedings, Vol. 2, 1994, pp 856-863

[18]R. Asensi, J.A. Cobos, O. Garcia, R. Prieto \& J Uceda, A CAD tool for magnetic components modeling, IEEE Applied Power Electronics Conference and Exposition APEC, Vol. 1, 1996, pp 427-433

[19]R. Prieto, J.A. Cobos, O. Garcia, P. Alou \& J. Uceda, "Taking into account all the parasitic effects in the design of magnetic components", APEC '98, Vol. 1, 1998, pp400-406 
[20]R. Prieto, J.A. Cobos, O. Garcia \& J. Uceda, "Interleaving techniques in magnetic components", APEC '97, Vol. 2, pp931-936

[21]R. Prieto, J.A. Cobos, O. Garcia \& J. Uceda, "Influence of the winding strategy on the parasitics of magnetic components", Proceedings EPE '97, Vol. 2, pp38-43

[22] Okyere P.F. \& Heinemann L., "An Advanced SpiceCompatible Model for High Frequency Multiwinding Transformers", IEEE Annual Power Electronics Specialists Conference (PESC), Vol. 1, 1999, pp 592597

[23] Evans P.D., Heffernan W.J.B., "Transformer for multimegahertz power applications", IEE Proceedings on Electric Power Applications, Vol. 142, No. 6, November 1995, pp379-389

[24]E.C. Cherry, "The duality between inter-linked electric and magnetic circuits", Proceedings of the Physics Society, Volume 62, 1949 pp101-111

[25]E. R. Laithwaite, "Magnetic Equivalent Circuits for electrical machines", PROC. IEE, Vol. 114, November 1967, pp1805-1809

[26] J. M. Lopera, A. M. Pernía, J. Díaz, J. M. Alonso and F. Nuño, "A complete transformer Electric Model, including Frequency and Geometric Effects", IEEE Power Electronics Specialists Conference, Toledo (Spain), June-July 1992, pp1247-1252

[27] A. M. Pernía, F. Nuño and J. M. Lopera, "Magnetic Elements Simulation in Power Converters", Congreso Internacional de Electrónica de Potencia, Puebla (Mexico), Aug. 1994, pp74-79

[28]A. M. Pernía, F. Nuño and J. M. Lopera, "1D/2D Transformer Electric Model for Simulation in Power Converters", IEEE Power Electronics Specialists Conference, Atlanta (USA), June 1995, pp. 1043-1049

[29] M.J. Prieto, J.M Lopera, A.M. Pernía, F.F. Linera and F. Nuño, "High-Frequency magnetic elements design tool", IEEE Workshop on Computers in Power Electronics (COMPEL '98), Villa Erba (Italy), 1998, pp. 127-131 